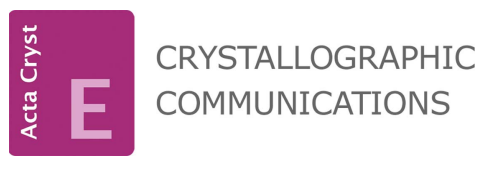

ISSN 2056-9890

Received 1 May 2020

Accepted 29 May 2020

Edited by S. Parkin, University of Kentucky, USA

Keywords: crystal structure; copper(II); polymer; tetramethylethylenediamine; Hirshfeld surface.

CCDC reference: 2006634

Supporting information: this article has supporting information at journals.iucr.org/e

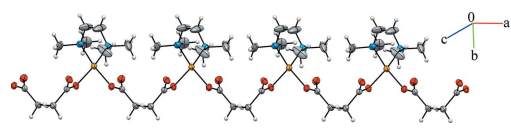

\section{Crystal structure and Hirshfeld surface analysis of one-dimensional copper(II) coordination polymer incorporating succinate and tetramethylethylene- diamine ligands}

\author{
Adnan M. Qadir, ${ }^{a}$ Sevgi Kansiz, ${ }^{b_{*}}$ Necmi Dege, ${ }^{c}$ Georgina M. Rosair ${ }^{d}$ and \\ Turganbay S. Iskenderov ${ }^{\mathbf{e}}$ *
}

${ }^{\mathbf{a} D}$ Department of Chemistry, College of Science, Salahaddin University, Erbil, 44001, Iraq, ${ }^{\mathbf{b}}$ Department of Fundamental
Sciences, Faculty of Engineering, Samsun University, Samsun, 55420, Turkey, ${ }^{\mathbf{c} D e p a r t m e n t ~ o f ~ P h y s i c s, ~ F a c u l t y ~ o f ~ A r t s ~ a n d ~}$
Sciences, Ondokuz Mayıs University, Samsun, 55139, Turkey, ' Institute of Chemical Sciences, School of Engineering \&
Physical Sciences, Heriot-Watt University, Edinburgh, EH14 4AS, UK, and ${ }^{\mathbf{d}}$ Department of Chemistry, National Taras
Shevchenko University of Kyiv, 64, Vladimirska Str., Kiev 01601, Ukraine. *Correspondence e-mail:
sevgi.kansiz85@gmail.com, tiskenderov@ukr.net

The reaction of copper nitrate with succinic acid (succH) and $N, N, N^{\prime}, N^{\prime}$ tetramethylethylenediamine (TMEDA) in basic solution produces the complex catena-poly[[[ $\left(N, N, N^{\prime}, N^{\prime}\right.$-tetramethylethylenediamine- $\left.\left.\kappa^{2} N, N^{\prime}\right) \operatorname{copper}(\mathrm{II})\right]-\mu$ succinato- $\left.\kappa^{2} O^{1}: O^{4}\right]$ tetrahydrate], $\left\{\left[\mathrm{Cu}\left(\mathrm{C}_{4} \mathrm{H}_{4} \mathrm{O}_{4}\right)\left(\mathrm{C}_{6} \mathrm{H}_{16} \mathrm{~N}_{2}\right)\right] \cdot 4 \mathrm{H}_{2} \mathrm{O}\right\}_{n}$ or $\left\{[\mathrm{Cu}(\text { succ })(\text { tmeda })] \cdot 4 \mathrm{H}_{2} \mathrm{O}\right\}_{n}$. Each carboxylate group of the succinate ligand coordinates to $\mathrm{a} \mathrm{Cu}^{\mathrm{II}}$ atom in a monodentate fashion, giving rise to a distorted square-planar geometry. The succinate ligands bridge the $\mathrm{Cu}^{\mathrm{II}}$ centres, forming one-dimensional polymeric chains. Hydrogen bonds between the ligands and water molecules link these chains into sheets that lie parallel to the $a c$ plane. Hirshfeld surface analysis, $d_{\text {norm }}$ and two-dimensional fingerprint plots were examined to verify the contributions of the different intermolecular contacts within the supramolecular structure.

\section{Chemical context}

Coordination polymers are a key area of development in supramolecular chemistry. Aliphatic saturated dicarboxylates are versatile linkage ligands for construction of supramolecular frameworks. These possess conformational freedom and coordinating ability owing to the single carbon chain. Aliphatic dicarboxylate anions exhibit different coordination modes such as uni-bidentate, bis-monodentate, bis-bidentate, tridentate or tetradentate, linking metal atoms into 1-D coordination polymers, 2-D layers or 3-D networks. Copper(II) carboxylate complexes are known to possess various biological activities including antifungal (Melník et al., 1982), antibacterial (Mojumdar et al., 2005), antiviral and cytotoxic activities (Ranford et al., 1993). Copper(II) is present at the active site some of proteins. The proteins containing copper(II) display biological functions such as electron transfer, dioxygen transfer, oxygenation, reduction, oxidation and disproportionation (Mukherjee, 2003). In this work, the synthesis, single crystal structure and Hirshfeld surface analysis of a copper(II) complex involving $N, N, N^{\prime}, N^{\prime}$ tetramethylethylenediamine and succinate ligands are reported. 
<smiles>COC1(OC(=O)CCC(=O)O)C(C)(C)CC[N+]1(C)C</smiles>

\section{Structural commentary}

The asymmetric unit of the title compound (1) is illustrated in Fig. 1. In the complex $\left\{\left[\mathrm{Cu}(\text { succ })_{2}(\text { tmeda })\right] \cdot 4 \mathrm{H}_{2} \mathrm{O}\right\}_{n}$, the central metal atom has distorted square-planar geometry with one oxygen atom each from two succ ligands and two TMEDA ligand nitrogen atoms (Figs. 2 and 3). There are two longer axial $\mathrm{Cu} \cdots \mathrm{O}$ contacts of $2.590(2)$ and $2.432(2) \AA$. In the square-plane, the $\mathrm{Cu}-\mathrm{O}$ and $\mathrm{Cu}-\mathrm{N}$ bond lengths are in the range 1.964 (2)-2.038 (2) $\AA$ (Table 1). The structural parameters in the TMEDA ligand, i.e. the $\mathrm{Cu}-\mathrm{N}$ bond lengths, are in agreement with those reported for the $\left[\mathrm{Cu}_{3}(\mathrm{PyDHA}\right.$ $\left.2 \mathrm{H})(\text { tmeda })_{3}\right]\left(\mathrm{ClO}_{4}\right)_{2}$ complex $($ PyDHA $=$ pyridine-2,6-dihydroxamic acid) by Gumienna-Kontecka et al. (2013). Similar geometric parameters have also been reported for $\left\{[\mathrm{Cu} \text { (succ)(deed) }] \cdot 4 \mathrm{H}_{2} \mathrm{O}\right\}_{n} \quad[\mathrm{Cu}-\mathrm{O}: \quad 2.123(8)-2.142(8) \AA$ deed $=N, N$-diethylethylenediamine; Şen et al., 2017] and $\left[\mathrm{Cu}_{2}\left(\mathrm{C}_{4} \mathrm{H}_{4} \mathrm{O}_{4}\right)_{2}\left(\mathrm{C}_{12} \mathrm{H}_{12} \mathrm{~N}_{2}\right)\right]_{n}$, [Cu-O: 1.955 (4)-1.983 (5) $\AA$; González Garmendia et al., 2009]. Selected bond lengths and

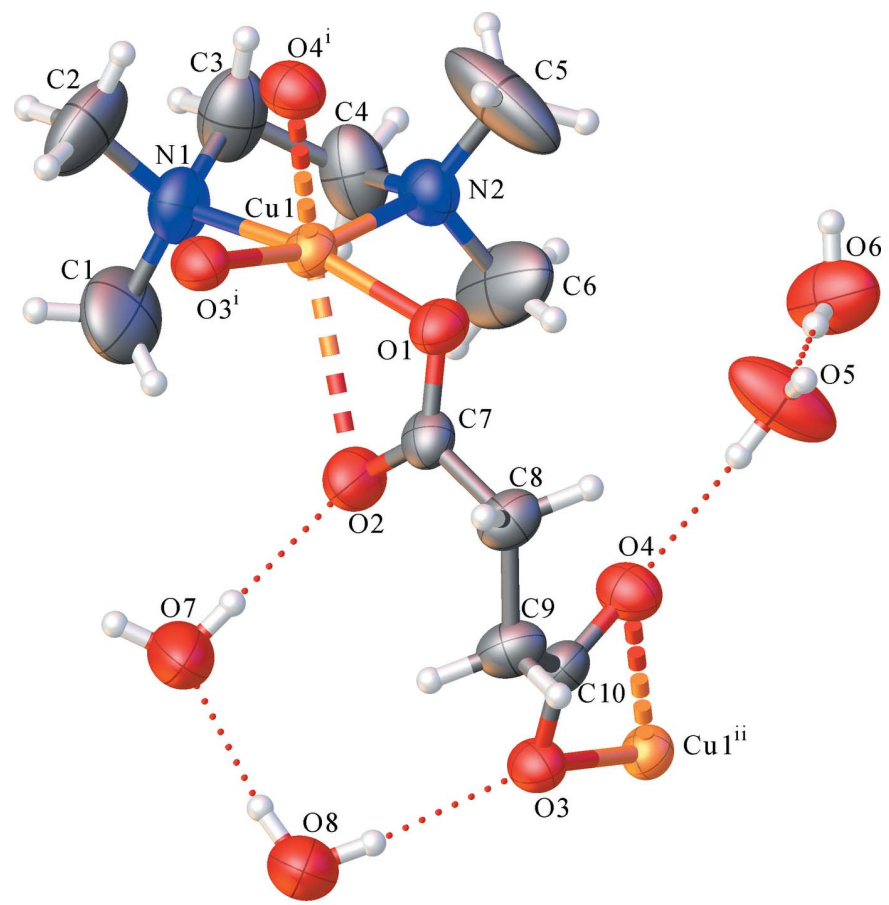

Figure 1

Perspective view of $\left\{[\mathrm{Cu} \text { (succ) (tmeda) }] \cdot 4 \mathrm{H}_{2} \mathrm{O}\right\}_{n}$, with the atom labelling. Displacement ellipsoids are drawn at the $50 \%$ probability level [symmetry codes: (i) $-1+x, y, z$; (ii) $1+x, y, z$ ].
Table 1

Selected geometric parameters $\left(\AA{ }^{\circ}\right)$.

\begin{tabular}{lclr}
\hline $\mathrm{Cu} 1-\mathrm{O} 1$ & $1.9639(17)$ & $\mathrm{O} 1-\mathrm{C} 7$ & $1.275(3)$ \\
$\mathrm{Cu} 1-\mathrm{O} 3^{\mathrm{i}}$ & $1.9958(16)$ & $\mathrm{O} 2-\mathrm{C} 7$ & $1.236(3)$ \\
$\mathrm{Cu} 1-\mathrm{O} 4^{\mathrm{i}}$ & $2.4315(17)$ & $\mathrm{O} 3-\mathrm{C} 10$ & $1.273(3)$ \\
$\mathrm{Cu} 1-\mathrm{N} 1$ & $2.024(2)$ & $\mathrm{O} 4-\mathrm{C} 10$ & $1.239(3)$ \\
$\mathrm{Cu} 1-\mathrm{N} 2$ & $2.038(2)$ & $\mathrm{N} 1-\mathrm{C} 1$ & $1.459(5)$ \\
& & & \\
$\mathrm{O} 1-\mathrm{Cu} 1-\mathrm{O}^{\mathrm{i}}$ & $89.80(7)$ & $\mathrm{O} 1-\mathrm{Cu} 1-\mathrm{N} 2$ & $92.40(8)$ \\
$\mathrm{O} 1-\mathrm{Cu} 1-\mathrm{O} 4^{\mathrm{i}}$ & $91.00(7)$ & $\mathrm{O}^{\mathrm{i}}-\mathrm{Cu} 1-\mathrm{N} 1$ & $94.20(8)$ \\
$\mathrm{O} 1-\mathrm{Cu} 1-\mathrm{N} 1$ & $167.77(9)$ & $\mathrm{O}^{\mathrm{i}}-\mathrm{Cu} 1-\mathrm{N} 2$ & $165.06(8)$ \\
\hline
\end{tabular}

Symmetry code: (i) $x-1, y, z$.

Table 2

Hydrogen-bond geometry $\left(\AA{ }^{\circ}\right)$.

\begin{tabular}{|c|c|c|c|c|}
\hline$D-\mathrm{H} \cdots A$ & $D-\mathrm{H}$ & $\mathrm{H} \cdots A$ & $D \cdots A$ & $D-\mathrm{H} \cdots A$ \\
\hline $\mathrm{O} 5-\mathrm{H} 5 \mathrm{H} \cdots \mathrm{O} 4$ & $0.79(2)$ & $1.98(2)$ & $2.763(3)$ & $169(6)$ \\
\hline $\mathrm{O} 6-\mathrm{H} 6 G \cdots \mathrm{O} 5$ & $0.82(2)$ & $1.88(2)$ & $2.694(3)$ & $175(5)$ \\
\hline $\mathrm{O} 7-\mathrm{H} 7 A \cdots \mathrm{O} 2$ & $0.82(2)$ & $1.96(2)$ & $2.774(3)$ & $172(6)$ \\
\hline $\mathrm{O} 8-\mathrm{H} 8 C \cdots \mathrm{O} 3$ & $0.83(2)$ & $2.04(2)$ & $2.869(3)$ & $173(5)$ \\
\hline $\mathrm{O} 8-\mathrm{H} 8 D \cdots \mathrm{O} 7$ & $0.83(2)$ & $1.93(2)$ & $2.733(4)$ & $165(5)$ \\
\hline $\mathrm{O} 7-\mathrm{H} 7 B \cdots \mathrm{O} 6^{\mathrm{ii}}$ & $0.81(1)$ & $1.97(2)$ & $2.763(4)$ & $167(6)$ \\
\hline $\mathrm{O} 5-\mathrm{H} 5 G \cdots \mathrm{O} 1^{\mathrm{iii}}$ & $0.80(2)$ & $2.00(2)$ & $2.799(3)$ & $176(6)$ \\
\hline $\mathrm{O} 6-\mathrm{H} 6 H \cdots \mathrm{O} 8^{\mathrm{iv}}$ & $0.84(2)$ & $2.01(2)$ & $2.803(4)$ & $157(5)$ \\
\hline
\end{tabular}

Symmetry codes: (ii) $\quad x-\frac{1}{2},-y+\frac{1}{2}, z+\frac{1}{2}$; (iii) $\quad-x+1,-y+1,-z+1$; (iv) $x-\frac{1}{2},-y+\frac{1}{2}, z-\frac{1}{2}$.

angles are given in Table 1. The succinate ligands bridge the $\mathrm{Cu}^{\mathrm{II}}$ centres, forming one-dimensional polymeric chains.

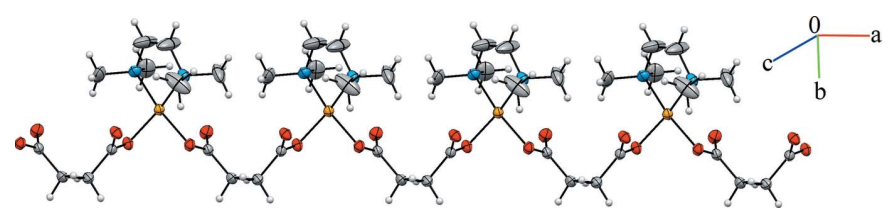

Figure 2

Ellipsoid plot $(50 \%)$ of a section of the polymeric chain of $\{[\mathrm{Cu}(\mathrm{succ})(\mathrm{t}-$ meda) $\left.] \cdot 4 \mathrm{H}_{2} \mathrm{O}\right\}_{n}$.

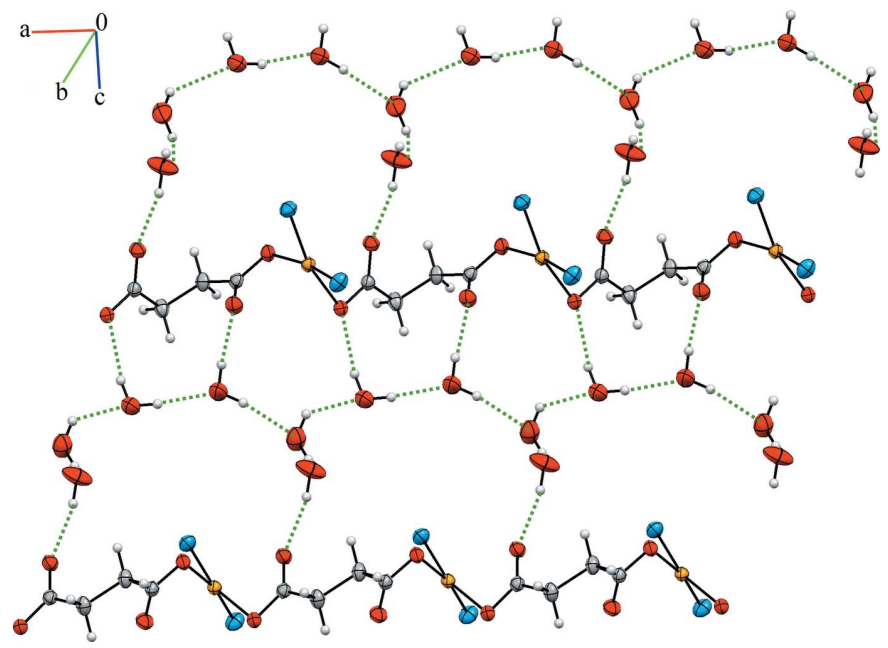

Figure 3

The two-dimensional layered structure of $\left\{\left[\mathrm{Cu}(\text { succ) }(\text { tmeda })] \cdot 4 \mathrm{H}_{2} \mathrm{O}\right\}_{n}\right.$. For clarity, the TMEDA ligands are shown only by their $\mathrm{N}$ atoms. 


\section{Supramolecular features}

In the asymmetric unit of the title complex, there are $\mathrm{O} 5-$ $\mathrm{H} 5 H \cdots \mathrm{O} 4, \quad \mathrm{O} 6-\mathrm{H} 6 G \cdots \mathrm{O} 5, \quad \mathrm{O} 7-\mathrm{H} 7 A \cdots \mathrm{O} 2, \quad \mathrm{O} 8-$ $\mathrm{H} 8 \mathrm{C} \cdots \mathrm{O} 3$ and $\mathrm{O} 8-\mathrm{H} 8 D \cdots \mathrm{O} 7$ hydrogen-bonding interactions, which act to stabilize the crystal packing. The crystal packing (Fig. 3) also features symmetry-related intermolecular hydrogen bonds $\left(\mathrm{O} 7-\mathrm{H} 7 B \cdots \mathrm{O} 6^{\mathrm{ii}}, \mathrm{O} 5-\mathrm{H} 5 G \cdots \mathrm{O} 1^{\mathrm{iii}}\right.$ and $\mathrm{O} 6-\mathrm{H} 6 H \cdots \mathrm{O}^{\mathrm{iv}}$; symmetry codes as in Table 2 ), linking the one-dimensional polymeric chains into sheets that lie parallel to the ac plane.

\section{Database survey}

A search of the Cambridge Structural Database (CSD, version 5.40, update of February 2019; Groom et al., 2016) for the title complex gave four hits: aqua(cyclobutane-1,1-dicarboxylato)$\left(N, N, N^{\prime}, N^{\prime}\right.$-tetramethylethylenediamine)copper(II) monohydrate (CBXECU; Pajunen \& Pajunen, 1979a), bis $\left(\mu_{2^{-}}\right.$ glutarato $)$ bis $\left[\left(N, N, N^{\prime}, N^{\prime}\right.\right.$-tetraethylethylenediamine $)$ copper(II)] (GLUECU; Pajunen \& Pajunen, 1979b), [N-(2oxybenzylidene)valinato $]\left(N, N, N^{\prime}, N^{\prime}\right.$-tetramethylethane-1,2diamine)copper(II) (UZAPES; Lakshmi et al., 2016) and $\left(N, N, N^{\prime}, N^{\prime \prime}, N^{\prime \prime}\right.$-pentamethyldiethylenetriamine)(L-valinato)copper(II) perchlorate (VEGRUU; Murakami \& Kita, 1998). The $\mathrm{Cu}-\mathrm{N}$ bond lengths range from 1.941 to $2.415 \AA$. When these bond lengths are compared with the title complex, the $\mathrm{Cu}-\mathrm{N}$ bond lengths $[2.024$ (2)-2.038 (2) $\AA$ ] fall within these limits.

\section{Hirshfeld surface analysis}

The Hirshfeld surface analysis (Spackman \& Jayatilaka, 2009) and the associated two-dimensional fingerprint plots were performed with CrystalExplorer17 (Turner et al., 2017).

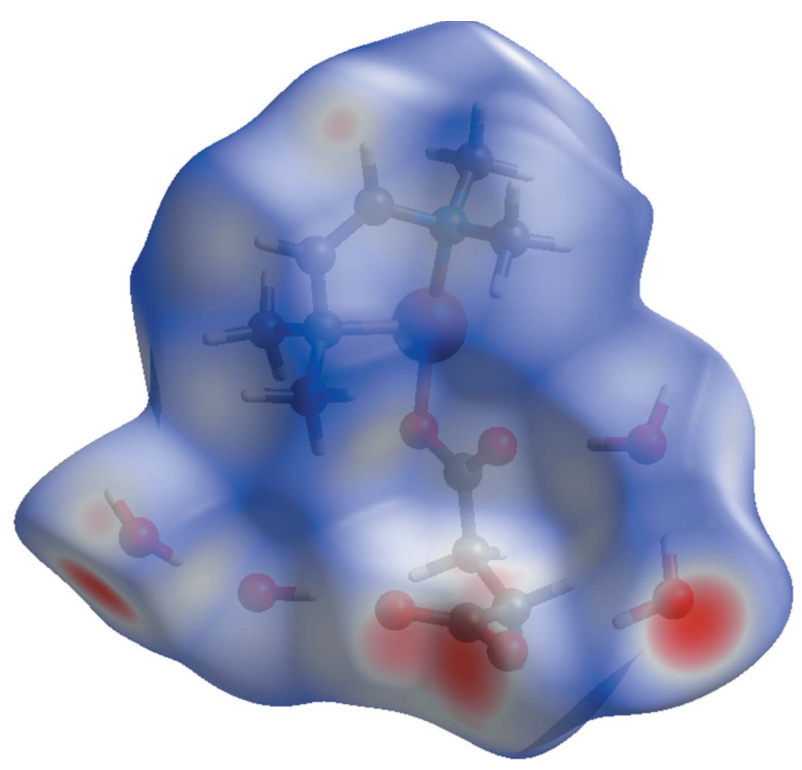

Figure 4

Hirshfeld surface mapped over $d_{\text {norm }}$ for $\left\{[\mathrm{Cu}(\text { succ })(\text { tmeda })] \cdot 4 \mathrm{H}_{2} \mathrm{O}\right\}_{n}$.
Hirshfeld surface analysis enables the visualization of intermolecular interactions by different colours and colour intensity, representing short or long contacts and indicating the relative strength of the interactions. Fig. 4 shows the Hirshfeld surface mapped over $d_{\text {norm }}$ ( -0.629 to 1.578 a.u.). The overall two-dimensional fingerprint plot for the title complex and those delineated into $\mathrm{H} \cdots \mathrm{H}, \mathrm{O} \cdots \mathrm{H} / \mathrm{H} \cdots \mathrm{O}$ and $\mathrm{Cu} \cdots \mathrm{O} /$ $\mathrm{O} \cdots \mathrm{Cu}$ contacts are illustrated in Fig. 5. The percentage contributions from the different inter-atomic contacts to the Hirshfeld surface are as follows: $\mathrm{H} \cdots \mathrm{H}(63.2 \%), \mathrm{O} \cdots \mathrm{H} /$ $\mathrm{H} \cdots \mathrm{O}(29.5 \%)$ and $\mathrm{Cu} \cdots \mathrm{O} / \mathrm{O} \cdots \mathrm{Cu}(3.8 \%)$. The percentage contributions for other intermolecular contacts amount to less than $3 \%$ of the Hirshfeld surface mapping.

\section{Synthesis and crystallization}

An aqueous solution of sodium succinate $(10 \mathrm{mmol}, 1.6 \mathrm{~g})$ was added to an aqueous solution of $\mathrm{Cu}\left(\mathrm{NO}_{3}\right)_{2} \cdot 3 \mathrm{H}_{2} \mathrm{O}(10 \mathrm{mmol}$, $2.4 \mathrm{~g}$ ) under stirring. A light-blue precipitate was formed. The precipitate was filtered and washed with water. The precipitate was dispersed in water and tetramethylethylenediamine $(10 \mathrm{mmol}, 1.2 \mathrm{~g})$ was added giving a dark-blue solution. The solution was filtered. Single crystals were obtained on slow evaporation of the solution after one week.

\section{Refinement}

Crystal data, data collection and structure refinement details are summarized in Table 3. Carbon-bound $\mathrm{H}$ atoms were
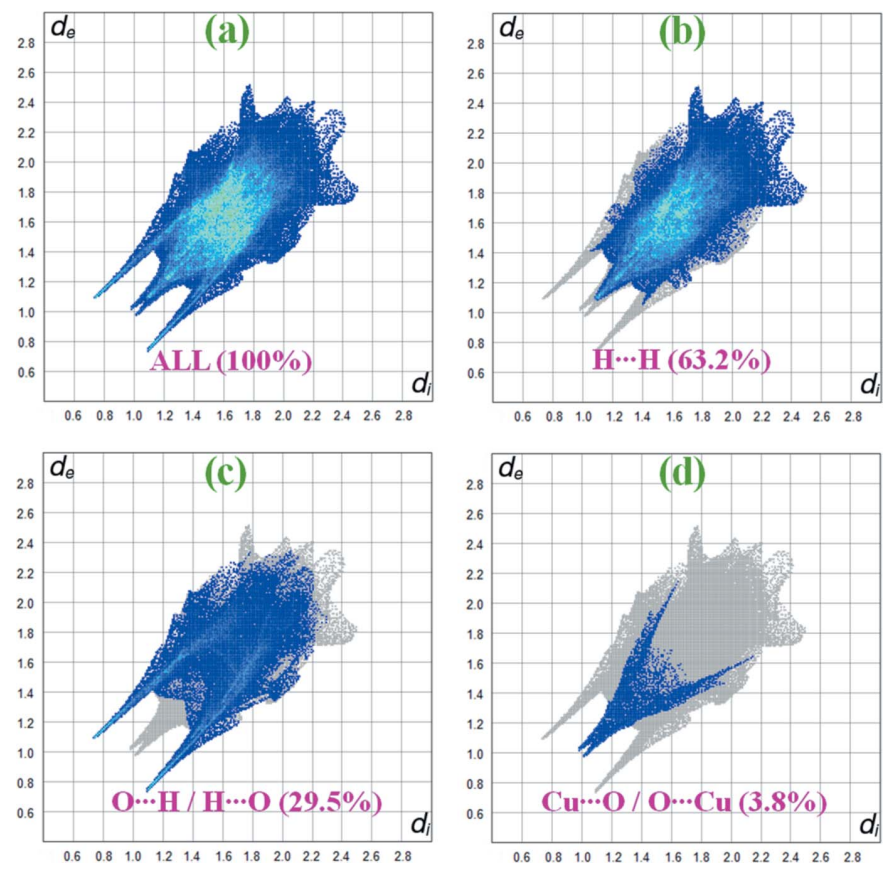

Figure 5

The two-dimensional fingerprint plots for $\left\{[\mathrm{Cu}(\text { succ })(\text { tmeda })] \cdot 4 \mathrm{H}_{2} \mathrm{O}\right\}_{n}$ showing the main interactions and their percentage contributions $\left(d_{\mathrm{i}}\right.$ is the closest internal distance from a given point on the Hirshfeld surface and $d_{\mathrm{e}}$ is the closest external contact). 
positioned geometrically and refined using a riding model, with $\mathrm{C}-\mathrm{H}=0.93,0.96$ and $0.97 \AA$ with $U_{\text {iso }}(\mathrm{H})=1.5 U_{\text {eq }}(\mathrm{C})$ for methyl $\mathrm{H}$ atoms and $1.2 U_{\text {eq }}(\mathrm{C})$ otherwise. The methyl groups were modelled as disordered over two torsional orientations. Water hydrogen-atom coordinates were refined, but $U_{\text {iso }}(\mathrm{H})$ was set to $1.5 U_{\text {eq }}$ (water $\left.\mathrm{O}\right)$.

\section{Funding information}

This study was supported by Ondokuz Mayıs University under project No. PYO·FEN.1906.19.001.

\section{References}

Dolomanov, O. V., Bourhis, L. J., Gildea, R. J., Howard, J. A. K. \& Puschmann, H. (2009). J. Appl. Cryst. 42, 339-341.

González Garmendia, M. J., Nacianceno, V. S., Seco, J. M. \& Zúñiga, F. J. (2009). Acta Cryst. C65, m436-m439.

Groom, C. R., Bruno, I. J., Lightfoot, M. P. \& Ward, S. C. (2016). Acta Cryst. B72, 171-179.

Gumienna-Kontecka, E., Golenya, I. A., Szebesczyk, A., Haukka, M., Krämer, R. \& Fritsky, I. O. (2013). Inorg. Chem. 52, 7633-7644.

Lakshmi, S. S., Geetha, K., Gayathri, M. \& Shanmugam, G. (2016). J. Chem. Sci. 128, 1095-1102.

Melník, M., Auderová, M. \& Hol'ko, M. (1982). Inorg. Chim. Acta, 67, 117-120.

Mojumdar, S. C., Madgurambal, G. \& Saleh, M. T. (2005). J. Therm. Anal. Calorim. 81, 205-210.

Mukherjee, R. N. (2003). Indian J. Chem. 42A, 2175-2184.

Murakami, T. \& Kita, S. (1998). Inorg. Chim. Acta, 274, 247-250.

Pajunen, A. \& Pajunen, S. (1979a). Acta Cryst. B35, 2401-2403.

Pajunen, A. \& Pajunen, S. (1979b). Acta Cryst. B35, 460-461.

Ranford, J. D., Sadler, P. J. \& Tocher, D. A. (1993). J. Chem. Soc. Dalton Trans. pp. 3393-3399.

Şen, F., Kansiz, S. \& Uçar, İ. (2017). Acta Cryst. C73, 517-524.

Sheldrick, G. M. (2015a). Acta Cryst. A71, 3-8.

Sheldrick, G. M. (2015b). Acta Cryst. C71, 3-8.

Spackman, M. A. \& Jayatilaka, D. (2009). CrystEngComm, 11, 19-32.

Stoe \& Cie (2002). $X$-AREA and $X$-RED32. Stoe \& Cie GmbH, Darmstadt, Germany.
Table 3

Experimental details.

\begin{tabular}{|c|c|}
\hline \multicolumn{2}{|l|}{ Crystal data } \\
\hline Chemical formula & {$\left[\mathrm{Cu}\left(\mathrm{C}_{4} \mathrm{H}_{4} \mathrm{O}_{4}\right)\left(\mathrm{C}_{6} \mathrm{H}_{16} \mathrm{~N}_{2}\right)\right] \cdot 4 \mathrm{H}_{2} \mathrm{O}$} \\
\hline$M_{\mathrm{r}}$ & 367.88 \\
\hline Crystal system, space group & Monoclinic, $P 2_{1} / n$ \\
\hline Temperature $(\mathrm{K})$ & 296 \\
\hline$a, b, c(\AA)$ & $\begin{array}{l}7.1195(4), 12.3172(6) \\
19.8590(12)\end{array}$ \\
\hline$\beta\left(^{\circ}\right)$ & $91.160(5)$ \\
\hline$V\left(\AA^{3}\right)$ & $1741.12(17)$ \\
\hline$Z$ & 4 \\
\hline Radiation type & Мо $K \alpha$ \\
\hline$\mu\left(\mathrm{mm}^{-1}\right)$ & 1.29 \\
\hline Crystal size $(\mathrm{mm})$ & $0.61 \times 0.33 \times 0.17$ \\
\hline \multicolumn{2}{|l|}{ Data collection } \\
\hline Diffractometer & Stoe IPDS 2 \\
\hline Absorption correction & $\begin{array}{l}\text { Integration }(X-R E D 32 \\
\quad \text { Stoe \& Cie, 2002) }\end{array}$ \\
\hline$T_{\min }, T_{\max }$ & $0.645,0.810$ \\
\hline $\begin{array}{l}\text { No. of measured, independent and } \\
\text { observed }[I>2 \sigma(I)] \text { reflections }\end{array}$ & $12278,3427,2864$ \\
\hline$R_{\text {int }}$ & 0.033 \\
\hline$(\sin \theta / \lambda)_{\max }\left(\AA^{-1}\right)$ & 0.617 \\
\hline \multicolumn{2}{|l|}{ Refinement } \\
\hline$R\left[F^{2}>2 \sigma\left(F^{2}\right)\right], w R\left(F^{2}\right), S$ & $0.034,0.091,1.04$ \\
\hline No. of reflections & 3427 \\
\hline No. of parameters & 233 \\
\hline No. of restraints & 10 \\
\hline $\mathrm{H}$-atom treatment & $\begin{array}{l}\mathrm{H} \text { atoms treated by a mixture of } \\
\text { independent and constrained } \\
\text { refinement }\end{array}$ \\
\hline$\Delta \rho_{\max }, \Delta \rho_{\min }\left(\mathrm{e} \AA^{-3}\right)$ & $0.28,-0.26$ \\
\hline
\end{tabular}

Computer programs: $X$-AREA and X-RED32 (Stoe \& Cie, 2002), SHELXT2017/1 (Sheldrick, 2015a), SHELXL2018/3 (Sheldrick, 2015b) and OLEX2 (Dolomanov et al., 2009).

Turner, M. J., MacKinnon, J. J., Wolff, S. K., Grimwood, D. J., Spackman, P. R., Jayatilaka, D. \& Spackman, M. A. (2017). Crystal Explorer17.5. University of Western Australia. http://hirshfeldsurface.net. 


\section{supporting information}

Acta Cryst. (2020). E76, 1038-1041［https://doi.org/10.1107/S2056989020007227]

Crystal structure and Hirshfeld surface analysis of one-dimensional copper(II) coordination polymer incorporating succinate and tetramethylethylenediamine ligands

Adnan M. Qadir, Sevgi Kansiz, Necmi Dege, Georgina M. Rosair and Turganbay S. Iskenderov

Computing details

Data collection: $X$-AREA (Stoe \& Cie, 2002); cell refinement: $X$-AREA (Stoe \& Cie, 2002); data reduction: X-RED32 (Stoe \& Cie, 2002); program(s) used to solve structure: SHELXT2017/1 (Sheldrick, 2015a); program(s) used to refine structure: SHELXL2018/3 (Sheldrick, 2015b); molecular graphics: OLEX2 (Dolomanov et al., 2009); software used to prepare material for publication: OLEX2 (Dolomanov et al., 2009).

catena-Poly $\left[\left[\left[\left(N, N, N^{\prime}, N^{\prime}\right.\right.\right.\right.$-tetramethylethylenediamine- $\left.\left.\kappa^{2} N, N^{\prime}\right) \operatorname{copper}(I I)\right]-\mu$-succinato- $\left.\kappa^{2} O^{1}: O^{4}\right]$ tetrahydrate $]$

Crystal data

$\left[\mathrm{Cu}\left(\mathrm{C}_{4} \mathrm{H}_{4} \mathrm{O}_{4}\right)\left(\mathrm{C}_{6} \mathrm{H}_{16} \mathrm{~N}_{2}\right)\right] \cdot 4 \mathrm{H}_{2} \mathrm{O}$

$M_{r}=367.88$

Monoclinic, $P 2{ }_{1} / n$

$a=7.1195(4) \AA$

$b=12.3172(6) \AA$

$c=19.8590(12) \AA$

$\beta=91.160(5)^{\circ}$

$V=1741.12(17) \AA^{3}$

$Z=4$

$F(000)=780$

$D_{\mathrm{x}}=1.403 \mathrm{Mg} \mathrm{m}^{-3}$

Mo $K \alpha$ radiation, $\lambda=0.71073 \AA$

Cell parameters from 19036 reflections

$\theta=1.7-29.9^{\circ}$

$\mu=1.29 \mathrm{~mm}^{-1}$

$T=296 \mathrm{~K}$

Stick, blue

$0.61 \times 0.33 \times 0.17 \mathrm{~mm}$

Data collection

Stoe IPDS 2

diffractometer

Detector resolution: 6.67 pixels $\mathrm{mm}^{-1}$

rotation method scans

Absorption correction: integration

(X-RED32; Stoe \& Cie, 2002)

$T_{\text {min }}=0.645, T_{\max }=0.810$

12278 measured reflections

3427 independent reflections

2864 reflections with $I>2 \sigma(I)$

$R_{\text {int }}=0.033$

$\theta_{\text {max }}=26.0^{\circ}, \theta_{\min }=2.0^{\circ}$

$h=-8 \rightarrow 8$

$k=-14 \rightarrow 15$

$l=-24 \rightarrow 24$

Hydrogen site location: mixed

$\mathrm{H}$ atoms treated by a mixture of independent and constrained refinement

$w=1 /\left[\sigma^{2}\left(F_{\mathrm{o}}^{2}\right)+(0.056 P)^{2}+0.2662 P\right]$

where $P=\left(F_{\mathrm{o}}{ }^{2}+2 F_{\mathrm{c}}{ }^{2}\right) / 3$

$(\Delta / \sigma)_{\max }=0.001$

$\Delta \rho_{\max }=0.28 \mathrm{e} \AA^{-3}$

$\Delta \rho_{\min }=-0.26$ e $\AA^{-3}$ 


\section{Special details}

Geometry. All esds (except the esd in the dihedral angle between two 1.s. planes) are estimated using the full covariance matrix. The cell esds are taken into account individually in the estimation of esds in distances, angles and torsion angles; correlations between esds in cell parameters are only used when they are defined by crystal symmetry. An approximate (isotropic) treatment of cell esds is used for estimating esds involving l.s. planes.

Fractional atomic coordinates and isotropic or equivalent isotropic displacement parameters $\left(\AA^{2}\right)$

\begin{tabular}{|c|c|c|c|c|c|}
\hline & $x$ & $y$ & $z$ & $U_{\text {iso }} * / U_{\text {eq }}$ & Occ. $(<1)$ \\
\hline $\mathrm{Cu} 1$ & $0.21554(4)$ & $0.28617(2)$ & $0.62467(2)$ & $0.03940(11)$ & \\
\hline $\mathrm{O} 1$ & $0.3640(2)$ & $0.41083(14)$ & $0.59461(8)$ & $0.0471(4)$ & \\
\hline $\mathrm{O} 2$ & $0.5331(3)$ & $0.34615(16)$ & $0.67869(10)$ & $0.0581(5)$ & \\
\hline $\mathrm{O} 3$ & $1.0718(2)$ & 0.38395 (14) & $0.68503(8)$ & $0.0457(4)$ & \\
\hline $\mathrm{O} 4$ & $0.9260(3)$ & 0.37395 (14) & $0.58714(8)$ & $0.0512(4)$ & \\
\hline $\mathrm{O} 7$ & $0.6289(4)$ & $0.3189(3)$ & $0.81373(13)$ & $0.1013(10)$ & \\
\hline $\mathrm{H} 7 \mathrm{~A}$ & $0.604(7)$ & $0.321(5)$ & $0.7735(10)$ & $0.152 *$ & \\
\hline $\mathrm{H} 7 \mathrm{~B}$ & $0.535(4)$ & $0.302(4)$ & $0.834(2)$ & $0.152^{*}$ & \\
\hline O8 & 0.9959 (4) & $0.3821(3)$ & $0.82634(12)$ & $0.0839(7)$ & \\
\hline $\mathrm{H} 8 \mathrm{C}$ & $1.023(7)$ & $0.387(4)$ & $0.7861(12)$ & $0.126^{*}$ & \\
\hline H8D & $0.892(4)$ & $0.353(4)$ & $0.826(3)$ & $0.126^{*}$ & \\
\hline N1 & $0.1142(4)$ & $0.14824(18)$ & $0.66575(12)$ & $0.0592(6)$ & \\
\hline $\mathrm{N} 2$ & $0.3026(3)$ & $0.19183(18)$ & $0.54703(11)$ & $0.0545(5)$ & \\
\hline $\mathrm{C} 1$ & $0.2261(7)$ & $0.1220(4)$ & $0.7259(2)$ & $0.1078(15)$ & \\
\hline H1A & 0.356188 & 0.117338 & 0.714512 & $0.129^{*}$ & 0.495 (18) \\
\hline H1B & 0.210105 & 0.177807 & 0.759095 & $0.129^{*}$ & 0.495 (18) \\
\hline $\mathrm{H} 1 \mathrm{C}$ & 0.185516 & 0.053701 & 0.743793 & $0.129 *$ & $0.495(18)$ \\
\hline H1D & 0.145018 & 0.115226 & 0.763755 & $0.129^{*}$ & $0.505(18)$ \\
\hline $\mathrm{H} 1 \mathrm{E}$ & 0.291101 & 0.054757 & 0.719171 & $0.129^{*}$ & $0.505(18)$ \\
\hline $\mathrm{H} 1 \mathrm{~F}$ & 0.315690 & 0.178863 & 0.734474 & $0.129^{*}$ & 0.505 (18) \\
\hline $\mathrm{C} 2$ & $-0.0839(5)$ & $0.1548(3)$ & 0.6849 (3) & $0.1029(15)$ & \\
\hline $\mathrm{H} 2 \mathrm{~A}$ & -0.160188 & 0.172027 & 0.645981 & $0.124^{*}$ & 0.505 \\
\hline $\mathrm{H} 2 \mathrm{~B}$ & -0.122672 & 0.086297 & 0.703029 & $0.124^{*}$ & 0.505 (18) \\
\hline $\mathrm{H} 2 \mathrm{C}$ & -0.098082 & 0.210404 & 0.718331 & $0.124 *$ & 0.505 (18) \\
\hline $\mathrm{H} 2 \mathrm{D}$ & -0.093773 & 0.140458 & 0.732246 & $0.124^{*}$ & 0.495 (18) \\
\hline $\mathrm{H} 2 \mathrm{E}$ & -0.131289 & 0.226188 & 0.675199 & $0.124 *$ & 0.495 (18) \\
\hline $\mathrm{H} 2 \mathrm{~F}$ & -0.155879 & 0.102082 & 0.659896 & $0.124^{*}$ & 0.495 (18) \\
\hline $\mathrm{C} 3$ & $0.086(3)$ & $0.0712(12)$ & $0.6087(8)$ & 0.085 & 0.495 (18) \\
\hline $\mathrm{H} 3 \mathrm{~A}$ & -0.018047 & 0.093743 & 0.579712 & $0.102 *$ & 0.495 (18) \\
\hline H3B & 0.062106 & -0.001667 & 0.624964 & $0.102 *$ & 0.495 (18) \\
\hline $\mathrm{C} 3 \mathrm{~A}$ & $0.179(2)$ & $0.0582(10)$ & $0.6193(8)$ & 0.077 (3) & 0.505 \\
\hline H3AA & 0.302001 & 0.033292 & 0.633975 & $0.093 *$ & 0.505 \\
\hline H3AB & 0.092507 & -0.002668 & 0.622077 & $0.093 *$ & 0.505 (18) \\
\hline $\mathrm{C} 4$ & $0.276(3)$ & $0.0761(8)$ & $0.5704(6)$ & $0.079(3)$ & $0.495(18)$ \\
\hline $\mathrm{H} 4 \mathrm{~A}$ & 0.378964 & 0.054535 & 0.600078 & $0.095^{*}$ & 0.495 (18) \\
\hline $\mathrm{H} 4 \mathrm{~B}$ & 0.271645 & 0.027282 & 0.532075 & $0.095^{*}$ & 0.495 (18) \\
\hline $\mathrm{C} 4 \mathrm{~A}$ & $0.187(2)$ & $0.0950(11)$ & $0.5496(7)$ & $0.087(4)$ & $0.505(18)$ \\
\hline H4AA & 0.239477 & 0.038474 & 0.521768 & $0.105^{*}$ & $0.505(18)$ \\
\hline $\mathrm{H} 4 \mathrm{AB}$ & 0.060916 & 0.111090 & 0.532601 & $0.105^{*}$ & $0.505(18)$ \\
\hline
\end{tabular}




$\begin{array}{llllll}\text { C5 } & 0.2202(9) & 0.2343(5) & 0.4859(2) & 0.144(3) & \\ \text { H5A } & 0.086425 & 0.238681 & 0.490305 & 0.173^{*} & 0.495(18) \\ \text { H5B } & 0.269870 & 0.305336 & 0.477526 & 0.173^{*} & 0.495(18) \\ \text { H5C } & 0.249344 & 0.187114 & 0.449077 & 0.173^{*} & 0.495(18) \\ \text { H5D } & 0.317334 & 0.248739 & 0.454300 & 0.173^{*} & 0.505(18) \\ \text { H5E } & 0.133889 & 0.182085 & 0.467080 & 0.173^{*} & 0.505(18) \\ \text { H5F } & 0.154415 & 0.300307 & 0.495528 & 0.173^{*} & 0.505(18) \\ \text { C6 } & 0.5040(6) & 0.1852(4) & 0.5403(3) & 0.1154(17) & \\ \text { H6A } & 0.559369 & 0.156878 & 0.581131 & 0.138^{*} & 0.505(18) \\ \text { H6B } & 0.533023 & 0.138122 & 0.503435 & 0.138^{*} & 0.505(18) \\ \text { H6C } & 0.553549 & 0.256345 & 0.531883 & 0.138^{*} & 0.505(18) \\ \text { H6D } & 0.537925 & 0.210685 & 0.496501 & 0.138^{*} & 0.495(18) \\ \text { H6E } & 0.564271 & 0.229441 & 0.574198 & 0.138^{*} & 0.495(18) \\ \text { H6F } & 0.543745 & 0.111219 & 0.545750 & 0.138^{*} & 0.495(18) \\ \text { C7 } & 0.5046(3) & 0.41647(19) & 0.63531(12) & 0.0424(5) & \\ \text { C8 } & 0.6324(4) & 0.51218(19) & 0.62714(14) & 0.0484(6) & \\ \text { H8A } & 0.566631 & 0.577064 & 0.641138 & 0.058^{*} & \\ \text { H8B } & 0.659682 & 0.520189 & 0.579733 & 0.058^{*} & \\ \text { C9 } & 0.8161(4) & 0.5050(2) & 0.66639(14) & 0.0510(6) & \\ \text { H9A } & 0.882166 & 0.573443 & 0.662250 & 0.061^{*} & \\ \text { H9B } & 0.789224 & 0.494519 & 0.713636 & 0.061^{*} & \\ \text { C10 } & 0.9432(3) & 0.41491(19) & 0.64394(12) & 0.0409(5) & \\ \text { O5 } & 0.7915(5) & 0.3966(2) & 0.45643(13) & 0.1012(10) & \\ \text { H5G } & 0.751(8) & 0.454(3) & 0.443(3) & 0.152^{*} & \\ \text { H5H } & 0.827(8) & 0.399(5) & 0.4946(13) & 0.152^{*} & \\ \text { O6 } & 0.8144(4) & 0.2050(2) & 0.39169(17) & 0.0906(8) & \\ \text { H6G } & 0.801(7) & 0.263(3) & 0.411(2) & 0.136^{*} & \\ \text { H6H } & 0.715(5) & 0.197(4) & 0.369(2) & 0.136^{*} & \end{array}$

Atomic displacement parameters $\left(\AA^{2}\right)$

\begin{tabular}{lllllll}
\hline & $U^{11}$ & $U^{22}$ & $U^{33}$ & $U^{12}$ & $U^{13}$ & $U^{23}$ \\
\hline Cu1 & $0.03491(16)$ & $0.04272(17)$ & $0.04064(16)$ & $0.00251(12)$ & $0.00232(10)$ & $0.00379(11)$ \\
O1 & $0.0359(9)$ & $0.0524(9)$ & $0.0529(9)$ & $0.0013(7)$ & $-0.0021(7)$ & $0.0085(7)$ \\
O2 & $0.0532(11)$ & $0.0621(11)$ & $0.0589(11)$ & $-0.0009(9)$ & $-0.0042(8)$ & $0.0186(9)$ \\
O3 & $0.0373(9)$ & $0.0555(10)$ & $0.0443(9)$ & $0.0048(7)$ & $-0.0012(7)$ & $-0.0012(7)$ \\
O4 & $0.0481(10)$ & $0.0573(10)$ & $0.0482(9)$ & $0.0097(8)$ & $-0.0044(7)$ & $-0.0052(8)$ \\
O7 & $0.0633(15)$ & $0.182(3)$ & $0.0581(14)$ & $-0.0182(18)$ & $-0.0042(11)$ & $0.0190(17)$ \\
O8 & $0.0686(15)$ & $0.124(2)$ & $0.0586(12)$ & $-0.0156(14)$ & $-0.0081(11)$ & $0.0052(14)$ \\
N1 & $0.0668(15)$ & $0.0447(11)$ & $0.0665(14)$ & $0.0005(11)$ & $0.0140(11)$ & $0.0083(10)$ \\
N2 & $0.0558(13)$ & $0.0586(13)$ & $0.0494(12)$ & $0.0057(10)$ & $0.0066(10)$ & $-0.0055(10)$ \\
C1 & $0.115(3)$ & $0.107(3)$ & $0.101(3)$ & $0.013(3)$ & $-0.002(2)$ & $0.059(3)$ \\
C2 & $0.068(2)$ & $0.078(2)$ & $0.164(4)$ & $-0.0142(19)$ & $0.028(2)$ & $0.037(3)$ \\
C3 & $0.101(10)$ & $0.061(6)$ & $0.095(7)$ & $-0.012(7)$ & $0.034(8)$ & $-0.005(5)$ \\
C3A & $0.076(7)$ & $0.045(4)$ & $0.112(8)$ & $0.000(5)$ & $0.020(7)$ & $0.004(4)$ \\
C4 & $0.116(10)$ & $0.055(4)$ & $0.069(6)$ & $0.017(5)$ & $0.026(6)$ & $-0.002(4)$ \\
C4A & $0.096(8)$ & $0.066(6)$ & $0.100(8)$ & $-0.023(5)$ & $0.011(6)$ & $-0.031(6)$ \\
C5 & $0.208(6)$ & $0.169(5)$ & $0.055(2)$ & $0.100(5)$ & $-0.035(3)$ & $-0.033(3)$
\end{tabular}




$\begin{array}{lllllll}\text { C6 } & 0.067(2) & 0.148(4) & 0.132(4) & 0.014(3) & 0.026(2) & -0.067(3) \\ \text { C7 } & 0.0361(12) & 0.0459(12) & 0.0453(12) & 0.0079(10) & 0.0072(9) & 0.0023(10) \\ \text { C8 } & 0.0395(13) & 0.0412(12) & 0.0646(15) & 0.0047(10) & 0.0023(11) & 0.0016(11) \\ \text { C9 } & 0.0414(13) & 0.0450(13) & 0.0664(16) & 0.0019(11) & -0.0021(11) & -0.0103(11) \\ \text { C10 } & 0.0337(12) & 0.0433(11) & 0.0459(12) & -0.0035(9) & 0.0034(9) & 0.0006(10) \\ \text { O5 } & 0.170(3) & 0.0614(13) & 0.0705(15) & 0.0345(16) & -0.0484(17) & -0.0091(12) \\ \text { O6 } & 0.0686(16) & 0.0971(19) & 0.106(2) & 0.0074(14) & 0.0017(13) & -0.0434(15)\end{array}$

Geometric parameters $\left(A,{ }^{\circ}\right)$

\begin{tabular}{|c|c|c|c|}
\hline $\mathrm{Cu} 1-\mathrm{O} 1$ & $1.9639(17)$ & $\mathrm{C} 3-\mathrm{H} 3 \mathrm{~A}$ & 0.9700 \\
\hline $\mathrm{Cu} 1-\mathrm{O}^{\mathrm{i}}$ & $1.9958(16)$ & $\mathrm{C} 3-\mathrm{H} 3 \mathrm{~B}$ & 0.9700 \\
\hline $\mathrm{Cu} 1-\mathrm{O} 4^{\mathrm{i}}$ & $2.4315(17)$ & $\mathrm{C} 3-\mathrm{C} 4$ & $1.56(2)$ \\
\hline $\mathrm{Cu} 1-\mathrm{N} 1$ & $2.024(2)$ & $\mathrm{C} 3 \mathrm{~A}-\mathrm{H} 3 \mathrm{AA}$ & 0.9700 \\
\hline $\mathrm{Cu} 1-\mathrm{N} 2$ & $2.038(2)$ & $\mathrm{C} 3 \mathrm{~A}-\mathrm{H} 3 \mathrm{AB}$ & 0.9700 \\
\hline $\mathrm{Cu} 1-\mathrm{C} 10^{\mathrm{i}}$ & $2.540(2)$ & $\mathrm{C} 3 \mathrm{~A}-\mathrm{C} 4 \mathrm{~A}$ & $1.46(2)$ \\
\hline $\mathrm{O} 1-\mathrm{C} 7$ & $1.275(3)$ & $\mathrm{C} 4-\mathrm{H} 4 \mathrm{~A}$ & 0.9700 \\
\hline $\mathrm{O} 2-\mathrm{C} 7$ & $1.236(3)$ & $\mathrm{C} 4-\mathrm{H} 4 \mathrm{~B}$ & 0.9700 \\
\hline $\mathrm{O} 3-\mathrm{C} 10$ & $1.273(3)$ & $\mathrm{C} 4 \mathrm{~A}-\mathrm{H} 4 \mathrm{AA}$ & 0.9700 \\
\hline $\mathrm{O} 4-\mathrm{C} 10$ & $1.239(3)$ & $\mathrm{C} 4 \mathrm{~A}-\mathrm{H} 4 \mathrm{AB}$ & 0.9700 \\
\hline $\mathrm{O} 7-\mathrm{H} 7 \mathrm{~A}$ & 0.815 (19) & $\mathrm{C} 5-\mathrm{H} 5 \mathrm{~A}$ & 0.9600 \\
\hline O7- & $0.812(10)$ & C5-H5B & 0.9600 \\
\hline $\mathrm{O} 8-\mathrm{H} 8 \mathrm{C}$ & 0.828 (19) & $\mathrm{C} 5-\mathrm{H} 5 \mathrm{C}$ & 0.9600 \\
\hline $\mathrm{O} 8-\mathrm{H} 8 \mathrm{D}$ & 0.827 (19) & C5-H5D & 0.9600 \\
\hline $\mathrm{N} 1-\mathrm{C} 1$ & $1.459(5)$ & C5-H5E & 0.9600 \\
\hline $\mathrm{N} 1-\mathrm{C} 2$ & $1.470(4)$ & $\mathrm{C} 5-\mathrm{H} 5 \mathrm{~F}$ & 0.9600 \\
\hline $\mathrm{N} 1-\mathrm{C} 3$ & $1.489(14)$ & C6-H6A & 0.9600 \\
\hline $\mathrm{N} 1-\mathrm{C} 3 \mathrm{~A}$ & $1.520(14)$ & C6-H6B & 0.9600 \\
\hline $\mathrm{N} 2-\mathrm{C} 4$ & $1.513(10)$ & C6- $\mathrm{H} 6 \mathrm{C}$ & 0.9600 \\
\hline $\mathrm{N} 2-\mathrm{C} 4 \mathrm{~A}$ & $1.453(11)$ & C6-H6D & 0.9600 \\
\hline $\mathrm{N} 2-\mathrm{C} 5$ & $1.436(5)$ & C6-H6E & 0.9600 \\
\hline N2-C6 & $1.445(4)$ & C6-H6F & 0.9600 \\
\hline $\mathrm{C} 1-\mathrm{H} 1 \mathrm{~A}$ & 0.9600 & $\mathrm{C} 7-\mathrm{C} 8$ & $1.500(3)$ \\
\hline $\mathrm{C} 1-\mathrm{H} 1 \mathrm{~B}$ & 0.9600 & $\mathrm{C} 8-\mathrm{H} 8 \mathrm{~A}$ & 0.9700 \\
\hline $\mathrm{C} 1-\mathrm{H} 1 \mathrm{C}$ & 0.9600 & $\mathrm{C} 8-\mathrm{H} 8 \mathrm{~B}$ & 0.9700 \\
\hline $\mathrm{C} 1-\mathrm{H} 1 \mathrm{D}$ & 0.9600 & $\mathrm{C} 8-\mathrm{C} 9$ & $1.512(3)$ \\
\hline $\mathrm{C} 1-\mathrm{H} 1 \mathrm{E}$ & 0.9600 & C9-H9A & 0.9700 \\
\hline $\mathrm{C} 1-\mathrm{H} 1 \mathrm{~F}$ & 0.9600 & C9-H9B & 0.9700 \\
\hline $\mathrm{C} 2-\mathrm{H} 2 \mathrm{~A}$ & 0.9600 & $\mathrm{C} 9-\mathrm{C} 10$ & $1.504(3)$ \\
\hline $\mathrm{C} 2-\mathrm{H} 2 \mathrm{~B}$ & 0.9600 & $\mathrm{O} 5-\mathrm{H} 5 \mathrm{G}$ & $0.80(2)$ \\
\hline $\mathrm{C} 2-\mathrm{H} 2 \mathrm{C}$ & 0.9600 & $\mathrm{O} 5-\mathrm{H} 5 \mathrm{H}$ & 0.794 (19) \\
\hline $\mathrm{C} 2-\mathrm{H} 2 \mathrm{D}$ & 0.9600 & O6- $\mathrm{H} 6 \mathrm{G}$ & $0.821(19)$ \\
\hline $\mathrm{C} 2-\mathrm{H} 2 \mathrm{E}$ & 0.9600 & $\mathrm{O} 6-\mathrm{H} 6 \mathrm{H}$ & 0.835 (19) \\
\hline $\mathrm{C} 2-\mathrm{H} 2 \mathrm{~F}$ & 0.9600 & & \\
\hline $\mathrm{O} 1-\mathrm{Cu} 1-\mathrm{O} 3^{\mathrm{i}}$ & $89.80(7)$ & $\mathrm{C} 4-\mathrm{C} 3-\mathrm{H} 3 \mathrm{~B}$ & 111.0 \\
\hline $\mathrm{O} 1-\mathrm{Cu} 1-\mathrm{O} 4^{\mathrm{i}}$ & $91.00(7)$ & $\mathrm{N} 1-\mathrm{C} 3 \mathrm{~A}-\mathrm{H} 3 \mathrm{AA}$ & 109.3 \\
\hline $\mathrm{O} 1-\mathrm{Cu} 1-\mathrm{N} 1$ & $167.77(9)$ & $\mathrm{N} 1-\mathrm{C} 3 \mathrm{~A}-\mathrm{H} 3 \mathrm{AB}$ & 109.3 \\
\hline
\end{tabular}




\begin{tabular}{|c|c|c|c|}
\hline $\mathrm{O} 1-\mathrm{Cu} 1-\mathrm{N} 2$ & $92.40(8)$ & $\mathrm{H} 3 \mathrm{AA}-\mathrm{C} 3 \mathrm{~A}-\mathrm{H} 3 \mathrm{AB}$ & 108.0 \\
\hline $\mathrm{O} 1-\mathrm{Cu} 1-\mathrm{C} 10^{\mathrm{i}}$ & $88.56(7)$ & $\mathrm{C} 4 \mathrm{~A}-\mathrm{C} 3 \mathrm{~A}-\mathrm{N} 1$ & $111.5(11)$ \\
\hline 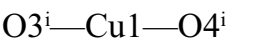 & $58.26(6)$ & $\mathrm{C} 4 \mathrm{~A}-\mathrm{C} 3 \mathrm{~A}-\mathrm{H} 3 \mathrm{AA}$ & 109.3 \\
\hline $\mathrm{O} 33^{\mathrm{i}}-\mathrm{Cu} 1-\mathrm{N} 1$ & $94.20(8)$ & $\mathrm{C} 4 \mathrm{~A}-\mathrm{C} 3 \mathrm{~A}-\mathrm{H} 3 \mathrm{AB}$ & 109.3 \\
\hline $\mathrm{O} 33^{\mathrm{i}}-\mathrm{Cu} 1-\mathrm{N} 2$ & $165.06(8)$ & $\mathrm{N} 2-\mathrm{C} 4-\mathrm{C} 3$ & $107.6(10)$ \\
\hline $\mathrm{O} 3^{\mathrm{i}}-\mathrm{Cu} 1-\mathrm{C} 10^{\mathrm{i}}$ & $29.61(7)$ & $\mathrm{N} 2-\mathrm{C} 4-\mathrm{H} 4 \mathrm{~A}$ & 110.2 \\
\hline 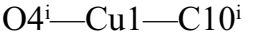 & $28.77(6)$ & $\mathrm{N} 2-\mathrm{C} 4-\mathrm{H} 4 \mathrm{~B}$ & 110.2 \\
\hline $\mathrm{N} 1-\mathrm{Cu} 1-\mathrm{O} 4^{\mathrm{i}}$ & $100.93(8)$ & $\mathrm{C} 3-\mathrm{C} 4-\mathrm{H} 4 \mathrm{~A}$ & 110.2 \\
\hline $\mathrm{N} 1-\mathrm{Cu} 1-\mathrm{N} 2$ & $86.72(9)$ & $\mathrm{C} 3-\mathrm{C} 4-\mathrm{H} 4 \mathrm{~B}$ & 110.2 \\
\hline $\mathrm{N} 1-\mathrm{Cu} 1-\mathrm{C} 10^{\mathrm{i}}$ & $100.59(9)$ & $\mathrm{H} 4 \mathrm{~A}-\mathrm{C} 4-\mathrm{H} 4 \mathrm{~B}$ & 108.5 \\
\hline $\mathrm{N} 2-\mathrm{Cu} 1-\mathrm{O} 4^{\mathrm{i}}$ & $106.90(8)$ & $\mathrm{N} 2-\mathrm{C} 4 \mathrm{~A}-\mathrm{C} 3 \mathrm{~A}$ & $108.8(11)$ \\
\hline $\mathrm{N} 2-\mathrm{Cu} 1-\mathrm{C} 10^{\mathrm{i}}$ & $135.64(9)$ & $\mathrm{N} 2-\mathrm{C} 4 \mathrm{~A}-\mathrm{H} 4 \mathrm{AA}$ & 109.9 \\
\hline $\mathrm{C} 7-\mathrm{O} 1-\mathrm{Cu} 1$ & $105.67(14)$ & $\mathrm{N} 2-\mathrm{C} 4 \mathrm{~A}-\mathrm{H} 4 \mathrm{AB}$ & 109.9 \\
\hline $\mathrm{C} 10-\mathrm{O} 3-\mathrm{Cu} 1^{\mathrm{ii}}$ & $99.60(14)$ & $\mathrm{C} 3 \mathrm{~A}-\mathrm{C} 4 \mathrm{~A}-\mathrm{H} 4 \mathrm{AA}$ & 109.9 \\
\hline $\mathrm{C} 10-\mathrm{O} 4-\mathrm{Cu} 1^{\mathrm{ii}}$ & $80.46(14)$ & $\mathrm{C} 3 \mathrm{~A}-\mathrm{C} 4 \mathrm{~A}-\mathrm{H} 4 \mathrm{AB}$ & 109.9 \\
\hline $\mathrm{H} 7 \mathrm{~A}-\mathrm{O} 7-\mathrm{H} 7 \mathrm{~B}$ & $109(2)$ & $\mathrm{H} 4 \mathrm{AA}-\mathrm{C} 4 \mathrm{~A}-\mathrm{H} 4 \mathrm{AB}$ & 108.3 \\
\hline $\mathrm{H} 8 \mathrm{C}-\mathrm{O} 8-\mathrm{H} 8 \mathrm{D}$ & $105(5)$ & $\mathrm{N} 2-\mathrm{C} 5-\mathrm{H} 5 \mathrm{~A}$ & 109.5 \\
\hline $\mathrm{C} 1-\mathrm{N} 1-\mathrm{Cu} 1$ & $108.8(2)$ & $\mathrm{N} 2-\mathrm{C} 5-\mathrm{H} 5 \mathrm{~B}$ & 109.5 \\
\hline $\mathrm{C} 1-\mathrm{N} 1-\mathrm{C} 2$ & $108.1(3)$ & $\mathrm{N} 2-\mathrm{C} 5-\mathrm{H} 5 \mathrm{C}$ & 109.5 \\
\hline $\mathrm{C} 1-\mathrm{N} 1-\mathrm{C} 3$ & $123.0(8)$ & $\mathrm{H} 5 \mathrm{~A}-\mathrm{C} 5-\mathrm{H} 5 \mathrm{~B}$ & 109.5 \\
\hline $\mathrm{C} 1-\mathrm{N} 1-\mathrm{C} 3 \mathrm{~A}$ & $99.7(6)$ & $\mathrm{H} 5 \mathrm{~A}-\mathrm{C} 5-\mathrm{H} 5 \mathrm{C}$ & 109.5 \\
\hline $\mathrm{C} 2-\mathrm{N} 1-\mathrm{Cu} 1$ & $114.2(2)$ & $\mathrm{H} 5 \mathrm{~B}-\mathrm{C} 5-\mathrm{H} 5 \mathrm{C}$ & 109.5 \\
\hline $\mathrm{C} 2-\mathrm{N} 1-\mathrm{C} 3$ & $96.8(7)$ & $\mathrm{H} 5 \mathrm{D}-\mathrm{C} 5-\mathrm{H} 5 \mathrm{E}$ & 109.5 \\
\hline $\mathrm{C} 2-\mathrm{N} 1-\mathrm{C} 3 \mathrm{~A}$ & $120.0(6)$ & $\mathrm{H} 5 \mathrm{D}-\mathrm{C} 5-\mathrm{H} 5 \mathrm{~F}$ & 109.5 \\
\hline $\mathrm{C} 3-\mathrm{N} 1-\mathrm{Cu} 1$ & $105.8(5)$ & $\mathrm{H} 5 \mathrm{E}-\mathrm{C} 5-\mathrm{H} 5 \mathrm{~F}$ & 109.5 \\
\hline $\mathrm{C} 3 \mathrm{~A}-\mathrm{N} 1-\mathrm{Cu} 1$ & $104.7(5)$ & $\mathrm{N} 2-\mathrm{C} 6-\mathrm{H} 6 \mathrm{~A}$ & 109.5 \\
\hline $\mathrm{C} 4-\mathrm{N} 2-\mathrm{Cu} 1$ & $105.2(4)$ & $\mathrm{N} 2-\mathrm{C} 6-\mathrm{H} 6 \mathrm{~B}$ & 109.5 \\
\hline $\mathrm{C} 4 \mathrm{~A}-\mathrm{N} 2-\mathrm{Cu} 1$ & $105.0(5)$ & $\mathrm{N} 2-\mathrm{C} 6-\mathrm{H} 6 \mathrm{C}$ & 109.5 \\
\hline $\mathrm{C} 5-\mathrm{N} 2-\mathrm{Cu} 1$ & $107.9(2)$ & $\mathrm{H} 6 \mathrm{~A}-\mathrm{C} 6-\mathrm{H} 6 \mathrm{~B}$ & 109.5 \\
\hline $\mathrm{C} 5-\mathrm{N} 2-\mathrm{C} 4$ & $123.4(8)$ & $\mathrm{H} 6 \mathrm{~A}-\mathrm{C} 6-\mathrm{H} 6 \mathrm{C}$ & 109.5 \\
\hline $\mathrm{C} 5-\mathrm{N} 2-\mathrm{C} 4 \mathrm{~A}$ & $96.1(8)$ & $\mathrm{H} 6 \mathrm{~B}-\mathrm{C} 6-\mathrm{H} 6 \mathrm{C}$ & 109.5 \\
\hline $\mathrm{C} 5-\mathrm{N} 2-\mathrm{C} 6$ & $109.4(4)$ & $\mathrm{H} 6 \mathrm{D}-\mathrm{C} 6-\mathrm{H} 6 \mathrm{E}$ & 109.5 \\
\hline $\mathrm{C} 6-\mathrm{N} 2-\mathrm{Cu} 1$ & $114.8(2)$ & $\mathrm{H} 6 \mathrm{D}-\mathrm{C} 6-\mathrm{H} 6 \mathrm{~F}$ & 109.5 \\
\hline $\mathrm{C} 6-\mathrm{N} 2-\mathrm{C} 4$ & $96.2(7)$ & $\mathrm{H} 6 \mathrm{E}-\mathrm{C} 6-\mathrm{H} 6 \mathrm{~F}$ & 109.5 \\
\hline $\mathrm{C} 6-\mathrm{N} 2-\mathrm{C} 4 \mathrm{~A}$ & $121.6(7)$ & $\mathrm{O} 1-\mathrm{C} 7-\mathrm{C} 8$ & $116.4(2)$ \\
\hline $\mathrm{N} 1-\mathrm{C} 1-\mathrm{H} 1 \mathrm{~A}$ & 109.5 & $\mathrm{O} 2-\mathrm{C} 7-\mathrm{O} 1$ & $121.3(2)$ \\
\hline $\mathrm{N} 1-\mathrm{C} 1-\mathrm{H} 1 \mathrm{~B}$ & 109.5 & $\mathrm{O} 2-\mathrm{C} 7-\mathrm{C} 8$ & $122.3(2)$ \\
\hline $\mathrm{N} 1-\mathrm{C} 1-\mathrm{H} 1 \mathrm{C}$ & 109.5 & $\mathrm{C} 7-\mathrm{C} 8-\mathrm{H} 8 \mathrm{~A}$ & 108.6 \\
\hline $\mathrm{H} 1 \mathrm{~A}-\mathrm{C} 1-\mathrm{H} 1 \mathrm{~B}$ & 109.5 & $\mathrm{C} 7-\mathrm{C} 8-\mathrm{H} 8 \mathrm{~B}$ & 108.6 \\
\hline $\mathrm{H} 1 \mathrm{~A}-\mathrm{C} 1-\mathrm{H} 1 \mathrm{C}$ & 109.5 & $\mathrm{C} 7-\mathrm{C} 8-\mathrm{C} 9$ & $114.7(2)$ \\
\hline $\mathrm{H} 1 \mathrm{~B}-\mathrm{C} 1-\mathrm{H} 1 \mathrm{C}$ & 109.5 & $\mathrm{H} 8 \mathrm{~A}-\mathrm{C} 8-\mathrm{H} 8 \mathrm{~B}$ & 107.6 \\
\hline $\mathrm{H} 1 \mathrm{D}-\mathrm{C} 1-\mathrm{H} 1 \mathrm{E}$ & 109.5 & $\mathrm{C} 9-\mathrm{C} 8-\mathrm{H} 8 \mathrm{~A}$ & 108.6 \\
\hline $\mathrm{H} 1 \mathrm{D}-\mathrm{C} 1-\mathrm{H} 1 \mathrm{~F}$ & 109.5 & $\mathrm{C} 9-\mathrm{C} 8-\mathrm{H} 8 \mathrm{~B}$ & 108.6 \\
\hline $\mathrm{H} 1 \mathrm{E}-\mathrm{C} 1-\mathrm{H} 1 \mathrm{~F}$ & 109.5 & $\mathrm{C} 8-\mathrm{C} 9-\mathrm{H} 9 \mathrm{~A}$ & 108.7 \\
\hline $\mathrm{N} 1-\mathrm{C} 2-\mathrm{H} 2 \mathrm{~A}$ & 109.5 & $\mathrm{C} 8-\mathrm{C} 9-\mathrm{H} 9 \mathrm{~B}$ & 108.7 \\
\hline $\mathrm{N} 1-\mathrm{C} 2-\mathrm{H} 2 \mathrm{~B}$ & 109.5 & $\mathrm{H} 9 \mathrm{~A}-\mathrm{C} 9-\mathrm{H} 9 \mathrm{~B}$ & 107.6 \\
\hline $\mathrm{N} 1-\mathrm{C} 2-\mathrm{H} 2 \mathrm{C}$ & 109.5 & $\mathrm{C} 10-\mathrm{C} 9-\mathrm{C} 8$ & $114.2(2)$ \\
\hline $\mathrm{H} 2 \mathrm{~A}-\mathrm{C} 2-\mathrm{H} 2 \mathrm{~B}$ & 109.5 & $\mathrm{C} 10-\mathrm{C} 9-\mathrm{H} 9 \mathrm{~A}$ & 108.7 \\
\hline
\end{tabular}




$\begin{array}{llll}\mathrm{H} 2 \mathrm{~A}-\mathrm{C} 2-\mathrm{H} 2 \mathrm{C} & 109.5 & \mathrm{C} 10-\mathrm{C} 9-\mathrm{H} 9 \mathrm{~B} & 108.7 \\ \mathrm{H} 2 \mathrm{~B}-\mathrm{C} 2-\mathrm{H} 2 \mathrm{C} & 109.5 & \mathrm{O} 3-\mathrm{C} 10-\mathrm{Cu} 1^{\mathrm{ii}} & 50.79(11) \\ \mathrm{H} 2 \mathrm{D}-\mathrm{C} 2-\mathrm{H} 2 \mathrm{E} & 109.5 & \mathrm{O} 3-\mathrm{C} 10-\mathrm{C} 9 & 117.4(2) \\ \mathrm{H} 2 \mathrm{D}-\mathrm{C} 2-\mathrm{H} 2 \mathrm{~F} & 109.5 & \mathrm{O} 4-\mathrm{C} 10-\mathrm{Cu} 1^{\mathrm{ii}} & 70.77(13) \\ \mathrm{H} 2 \mathrm{E}-\mathrm{C} 2-\mathrm{H} 2 \mathrm{~F} & 109.5 & \mathrm{O} 4-\mathrm{C} 10-\mathrm{O} 3 & 121.2(2) \\ \mathrm{N} 1-\mathrm{C} 3-\mathrm{H} 3 \mathrm{~A} & 111.0 & \mathrm{O} 4-\mathrm{C} 10-\mathrm{C} 9 & 121.4(2) \\ \mathrm{N} 1-\mathrm{C} 3-\mathrm{H} 3 \mathrm{~B} & 111.0 & \mathrm{C} 9-\mathrm{C} 10-\mathrm{Cu} 1^{\mathrm{ii}} & 166.01(17) \\ \mathrm{N} 1-\mathrm{C} 3-\mathrm{C} 4 & 104.0(13) & \mathrm{H} 5 \mathrm{G}-\mathrm{O} 5-\mathrm{H} 5 \mathrm{H} & 113(5) \\ \mathrm{H} 3 \mathrm{~A}-\mathrm{C} 3-\mathrm{H} 3 \mathrm{~B} & 109.0 & \mathrm{H} 6 \mathrm{G}-\mathrm{O} 6-\mathrm{H} 6 \mathrm{H} & 105(3) \\ \mathrm{C} 4-\mathrm{C} 3-\mathrm{H} 3 \mathrm{~A} & 111.0 & & \\ & & & 54.0(19) \\ \mathrm{Cu} 1-\mathrm{O} 1-\mathrm{C} 7-\mathrm{O} 2 & 5.8(3) & \mathrm{N} 1-\mathrm{C} 3 \mathrm{~A}-\mathrm{C} 4 \mathrm{~A}-\mathrm{N} 2 & -76.7(11) \\ \mathrm{Cu} 1-\mathrm{O} 1-\mathrm{C} 7-\mathrm{C} 8 & -174.58(16) & \mathrm{C} 1-\mathrm{N} 1-\mathrm{C} 3-\mathrm{C} 4 & -145.2(12) \\ \mathrm{Cu} 1{ }^{\mathrm{ii}}-\mathrm{O} 3-\mathrm{C} 10-\mathrm{O} 4 & 7.5(3) & \mathrm{C} 1-\mathrm{N} 1-\mathrm{C} 3 \mathrm{~A}-\mathrm{C} 4 \mathrm{~A} & 166.6(11) \\ \mathrm{Cu} 11^{\mathrm{ii}}-\mathrm{O} 3-\mathrm{C} 10-\mathrm{C} 9 & -171.00(18) & \mathrm{C} 2-\mathrm{N} 1-\mathrm{C} 3-\mathrm{C} 4 & 97.2(12) \\ \mathrm{Cu} 1{ }^{\mathrm{i}-\mathrm{O} 4-\mathrm{C} 10-\mathrm{O} 3} & -6.2(2) & \mathrm{C} 2-\mathrm{N} 1-\mathrm{C} 3 \mathrm{~A}-\mathrm{C} 4 \mathrm{~A} & -84.0(12) \\ \mathrm{Cu} 1{ }^{\mathrm{ii}}-\mathrm{O} 4-\mathrm{C} 10-\mathrm{C} 9 & 172.3(2) & \mathrm{C} 5-\mathrm{N} 2-\mathrm{C} 4-\mathrm{C} 3 & -155.7(13) \\ \mathrm{Cu} 1-\mathrm{N} 1-\mathrm{C} 3-\mathrm{C} 4 & 49.0(13) & \mathrm{C} 5-\mathrm{N} 2-\mathrm{C} 4 \mathrm{~A}-\mathrm{C} 3 \mathrm{~A} & 157.9(13) \\ \mathrm{Cu} 1-\mathrm{N} 1-\mathrm{C} 3 \mathrm{~A}-\mathrm{C} 4 \mathrm{~A} & -32.7(14) & \mathrm{C} 6-\mathrm{N} 2-\mathrm{C} 4-\mathrm{C} 3 & 87.1(12) \\ \mathrm{Cu} 1-\mathrm{N} 2-\mathrm{C} 4-\mathrm{C} 3 & 40.1(15) & \mathrm{C} 6-\mathrm{N} 2-\mathrm{C} 4 \mathrm{~A}-\mathrm{C} 3 \mathrm{~A} & 65.1(3) \\ \mathrm{Cu} 1-\mathrm{N} 2-\mathrm{C} 4 \mathrm{~A}-\mathrm{C} 3 \mathrm{~A} & -45.4(15) & \mathrm{C} 7-\mathrm{C} 8-\mathrm{C} 9-\mathrm{C} 10 & 169.4(6) \\ \mathrm{O} 1-\mathrm{C} 7-\mathrm{C} 8-\mathrm{C} 9 & -168.5(2) & \mathrm{C} 8-\mathrm{C} 9-\mathrm{C} 10-\mathrm{Cu} 1^{\mathrm{ii}} & -160.5(2) \\ \mathrm{O} 2-\mathrm{C} 7-\mathrm{C} 8-\mathrm{C} 9 & 11.1(3) & \mathrm{C} 8-\mathrm{C} 9-\mathrm{C} 10-\mathrm{O} 3 & 20.9(3) \\ \mathrm{N} 1-\mathrm{C} 3-\mathrm{C} 4-\mathrm{N} 2 & -60.5(18) & \mathrm{C} 8-\mathrm{C} 9-\mathrm{C} 10-\mathrm{O} 4 & \\ & & & \end{array}$

Symmetry codes: (i) $x-1, y, z$; (ii) $x+1, y, z$.

Hydrogen-bond geometry $\left(\AA,{ }^{\circ}\right)$

\begin{tabular}{|c|c|c|c|c|}
\hline$D-\mathrm{H} \cdots A$ & $D-\mathrm{H}$ & $\mathrm{H} \cdots A$ & $D \cdots A$ & $D-\mathrm{H} \cdots A$ \\
\hline $\mathrm{O} 5-\mathrm{H} 5 H \cdots \mathrm{O} 4$ & $0.79(2)$ & $1.98(2)$ & $2.763(3)$ & $169(6)$ \\
\hline $\mathrm{O} 6-\mathrm{H} 6 G^{\cdots} \cdots \mathrm{O} 5$ & $0.82(2)$ & $1.88(2)$ & $2.694(3)$ & $175(5)$ \\
\hline $\mathrm{O} 7-\mathrm{H} 7 A \cdots \mathrm{O} 2$ & $0.82(2)$ & $1.96(2)$ & $2.774(3)$ & $172(6)$ \\
\hline $\mathrm{O} 8-\mathrm{H} 8 C \cdots \mathrm{O} 3$ & $0.83(2)$ & $2.04(2)$ & $2.869(3)$ & $173(5)$ \\
\hline $\mathrm{O} 8-\mathrm{H} 8 D^{\cdots \mathrm{O}} 7$ & $0.83(2)$ & $1.93(2)$ & $2.733(4)$ & $165(5)$ \\
\hline $\mathrm{O} 7-\mathrm{H} 7 B^{\cdots} \mathrm{O}^{\mathrm{iii}}$ & $0.81(1)$ & $1.97(2)$ & $2.763(4)$ & $167(6)$ \\
\hline $\mathrm{O} 5-\mathrm{H} 5 G^{\cdots} \mathrm{O} 1^{\mathrm{iv}}$ & $0.80(2)$ & $2.00(2)$ & $2.799(3)$ & $176(6)$ \\
\hline $\mathrm{O} 6-\mathrm{H} 6 H^{\cdots} \mathrm{O} 8^{v}$ & $0.84(2)$ & $2.01(2)$ & $2.803(4)$ & $157(5)$ \\
\hline
\end{tabular}

Symmetry codes: (iii) $x-1 / 2,-y+1 / 2, z+1 / 2$; (iv) $-x+1,-y+1,-z+1$; (v) $x-1 / 2,-y+1 / 2, z-1 / 2$. 